\title{
THE USE OF STANDARDIZED ASSESSMENT TESTS IN THE PROCESS OF SPEECH THERAPY INTERVENTION
}

\author{
AUTHOR'S DATA: \\ Anna Malkhasyan, Researcher \\ Chair of Speech and Rehabilitative Therapy, Khachatur Abovyan Armenian State Pedagogical University \\ Applicant, researcher \\ Speech therapist in "Madatyan Psychological Center" \\ Contacts: anna-malkhasyan@mail.ru
}

\section{ABSTRACT}

To study and comment the importance of speech research process in the speech therapeutic correctional process, to discover how the objectivity of speech evaluation process is providing in Republic of Armenia. The process of studying, discovering and commenting the objectivity and the importance of speech research in Republic of Armenia will help to organize the first research stage of correcting and developing the speech. The experience shows, that the level of efficiency of speech correctional developing work generally depends on objectivity of research stage, the speech evaluation, and the reliability of data obtained.

The methodology of the research of gathering information, processing and analyzing is based on quantitative method approach, which allows to combine quantitative data collection by using quantitative method, subsequently, having opportunity to make data based conclusions, based on digital patterns. The special developed test was used for speech therapists and for the relating field specialists (psychologists, special educators, art therapists, and etc.).

The research has shown, that speech therapists and other specialists of the relating fields of Republic of Armenia attach importance of speech assessment process; the majority of respondents have his/her own speech evaluation tools, methods, and resources, however, none of them uses standardized test in order to highlight the features of speech, and, unfortunately, they state that their evaluation is not objective, but subjective and it may be questioned by other specialists and parents. However, it should also be stated that respondents unanimously noted, that there was a need of having speech assessment standardized tests, which would be relevant to the native language and as a result of test application objective results will be known. 
Key words: Speech Assessment, Speech Therapy, Standardized Tests, International Classification of Function, children with speech disorders.

\section{INTRODUCTION}

Based on Republic of Armenia (RA) Government protocol decision of Session N1 on January 9, 2014, “A person's comprehensive evaluation of World Health Organization International Classification of Functioning based on Implementation model of defining disability (hereinafter WHO ICF-CY)" according to which in recent years, in Republic of Armenia, in the scope of the social model holding of defining disability, improvements are carrying out not only in the area of defining disability but also in areas of disability evaluation, disability grade provision, service creation and provision, monitoring, and evaluation (Government of the Republic of Armenia 2014 Annex N 1 to the Protocol on the January 9 Session Decision Protocol Annex 1: Introduction of a Model for Defining Disability Based on the International Classification of Functions of the World Health Organization).

The World Health Organization International Classification of Function Children and Youth (WHO ICF-CY) allows the use of common language and terminology, thereby recording the problems in organ system or in its functions, limitations of person's activity and participation, as well as the impact of corresponding environmental factors in the period of neonatal, childhood and adolescence (WHO ICF-CY, 2001). The WHO ICF-CY allows to use one common language in the areas of healthcare, education, and social protection and to create conditions for using the available opportunities for the benefit of children and teenagers with developmental delay or disabilities. Children's and teenagers' health problems differ from adults' disabilities and health problems by its nature, way of expression and impact. Taking into account these differences, the classification was compiled in such a way that it became possible completely describe changes connecting with development. Therefore, it allows to involve different age groups and different environments features (ALTE, 2014).

From June 2016 Functional Assessment Methodological Handbook was developing by several stages, first, the codes were selected by the specialists, then the codes qualifiers were established, after which the Methodological Handbook was put into practice from a period of June - August (for 3 months), during this period 50 children were evaluated. During the usage changes were made, some codes were removed, some qualifiers were clarified. The second trial started from September 2016 (Avetisyan, Ghazaryan, Malkhasyan, 2017). It lasted 6 months. Overall, 100 
children were assessed, after which some qualifiers were also clarified and modified. About 200 beneficiaries attending to "Full Life" Non-Governmental Organization (NGO) Centers of Stepanavan and Yerevan were evaluated by the evaluation tools of 2016. From 2 to 18 years old children with disabilities and children with some problems without disability status were evaluated.

In 2017-2018, a new stage of development of Methodological Handbook was launched, as a result both content and structural changes were made, and particularly new codes were added, corresponding qualifiers were developed, changes were made as in the structure of the test as in usage of it during the evaluation. Individual plan forms of intervention were developed for the multidisciplinary team members. At the same time ICF tools were spread and used in other NGOs, as a result, today about 12 NGOs use ICF tools with totally 431 children with disabilities in their centers. Based on the whole results and conclusions of the process, WHO ICF-CY approach attaches importance and it attempts to be invested in the basis of activity, participation, and environmental factors evaluation of the Armenian children and teenagers with the need of rehabilitation services. Thus, as a result of person's comprehensive evaluation, the proposed tools allow to develop individual programs of development, according to his/her personal abilities and needs, and to provide appropriate services to promote children and teenagers with disability or psychophysical disorder for their activity, participation, and for living independently and for involving in community life.

The notable is the fact, that being involved in the process of training the professional teams of the 12 NGOs, it was established that almost the majority of the specialists noted that they did not have enough tools, resources and methods which would promote to get objective, reliable and trustworthy information as a result of speech research. So we have examined the international experience, that is, the experience of the countries where functional evaluation is already applied, as they already had experience in this field. The research of the speech development problem is relevance as the native language plays a special role in the formation of the child's personality. In psychology, philosophy, and pedagogy speech and language traditionally viewed as "knot", where the different lines of mental development - thinking, imagination, memory, and emotions are intersected.

Early age is the more important period for the development of speech. Speech disorders make difficulties in the communication with close adults, impede the development of cognitive 
processes, and have a negative impact on the formation of self-consciousness. This is the reason why the prevention of speech disorders is the current issues of the science (Gromova, 2003).

As speech disorders in early childhood can later affect vocabulary and formation of grammatical structures in the future, so for the prevention of secondary disorders complex speech therapeutic research is needed. Methodologies of speech studies of early age are observed in the works of Gromova, Pechrova, Chirkina, and Sheremeteva who had developed a test for early age children's speech investigation, which is filled by the parents (Gromova, 2003).

Standardized assessments are critically important in identifying language disorder in school-age children. They are vital because they accurately and reliably establish whether a child's language skills are developing as well as same age peers (Kaderavek, 2011).

Speech-language assessment is a complex process. Assessing, describing, and interpreting an individual's communication ability requires the integration of a variety of information gathered in the evaluation process. ASHA's (American Speech- Language- Hearing Association) Preferred Practice Patterns for the Professions of Speech-Language Pathology (SLP) (2004) indicates that comprehensive speech-language pathology assessment includes these components:

- Case history, including medical status, education, socioeconomic, cultural, and linguistic backgrounds and information from teachers and other related service providers

- Patient/client/student and family interview

- Review of auditory, visual, motor, and cognitive status

- Standardized and/or non-standardized measures of specific aspects of speech, spoken and non-spoken language, cognitive-communication, and swallowing function, including observations and analysis of work samples

- Identification of potential for effective intervention strategies and compensations

- Selection of standardized measures for speech, language, cognitive-communication, and/or swallowing assessment with consideration for documented ecological validity and cultural sensitivity

- Follow-up services to monitor communication and swallowing status and ensure appropriate intervention and support for individuals with identified speech, language, cognitive-communication, and/or swallowing disorders 
The Individuals with Disabilities Education Act (IDEA) has specific provisions concerning the assessment of students in schools. In addition, SLPs need to follow state and local requirements for the assessments of students (ALTE, 2014; Westby, Burda \& Mehta, 2003).

It is important to note the distinctions between terms evaluation and assessment according to IDEA Part C Guidelines. Evaluation means the "procedures used by qualified personnel to determine a child's initial and continuing eligibility...", Part B requires that an evaluation be comprehensive and assess all areas of suspected disability. It is important for the clinician to involve other assessment staff as part of the multidisciplinary evaluation team to address educational and/or behavioral concerns for students who are not meeting the grade-level expectations (ALTE, 2014). Assessment means "the ongoing procedures used by qualified personnel to identify the child's unique strengths and needs and the early intervention services appropriate to meet those needs throughout the period of the child's eligibility...and includes the assessment of the child...and the assessment of the child's family..." (IDEA, 2004).

Association of Language Testers in Europe (ALTE) is an association of language test providers who work together to promote the fair and accurate assessment of linguistic ability across Europe and beyond. ALTE organizes courses and conferences on aspects of language assessment, and operates a quality auditing system of European language examinations. ALTE was founded in 1989 by the University of Cambridge (UK) and the Universidad de Salamanca (Spain). Today, ALTE has 34 Full Members representing 25 European languages, as well as 58 Institutional Affiliates and over 500 Individual Affiliates from all around the world (WHO ICFCY, 2001).

Investigated studies have shown that European countries and the United States of America have more experience in this area and have tests, tools that promote the process of speech research (Gromova, 2003; Miqaelyan \& Miqaelyan, 2010). In order to obtain reliable data on to what extent was the importance of the process of speech assessment and investigation the research was carried out, to ensure the objectivity and reliability of that process and how trustworthy they were.

\section{LITERATURE REVIEW}

The word "test" came from ancient French and is a synonym for the word "cup" (Latin Testa - clay pot). This word was called small clay vials used for alchemy experiments. The word "test" in Russian had two meanings for a long time: 
1. Experimental oath, an English religious oath which every public official must give to prove that he is not a secret Catholic;

2. Flat floating bottle.

The test emerged in the early 20th century and it was applied in the fields of psychology, sociology and pedagogy. It has served to carry out some psychological or sociological research. Later American educators began to use the test as a way of testing students' knowledge, its perception, and application. In English the word test means experiment or check. In Armenian the word test used to refer another form of teaching task, which will be discussed below, so the foreign name of that checking form is keeping. The test is different from other forms of teaching and checking tasks, tests, textbook questions. The test is used as a fast, efficient, unbiased form of assessment that is suitable for testing and evaluating many people in a short period of time. Its role is especially enhanced when it comes to computer self-checking and self-monitoring.

Evaluation is a continuous, continuous process. It consists of quantitative and qualitative assessments. Assessment is the gathering of information about what level of learning the learner has achieved. Many valuation tools can be used to gather this information (Gromova, 2003). These may include numerous observations, written tests, homework, research, projects, oral inquiries, student-led record analysis. Written tests have their special place in them, and are one of the most actual effective ways of testing knowledge. Tests are characterized by the following points:

1. Coordination, the tasks involved are only about the same subject, even the scope of the same topic, and consist of simple to complex gradual development.

2. A special form, a test is not a set of questions or tasks. The task is usually formulated as a right or a wrong expression, which is conditioned by the response.

3. Logical task specificity: This means that the test task should not give rise to ambiguity or doubt; it has some positive or negative answer.

4. The answer to the test task is a brief judgment. At the same time, he chooses the correct answer from the ones already given. This is one of the important features of the test, the answer is already there, the respondent must choose from the given.

5. Has a special structure. There is a sequential link between the test tasks: each task stems from the previous one and further develops the topic at stake.

6. Success depends on experimentation. Another very important feature of the test task is that only after some testing can the test be confidently successful. 
There are a number of factors to consider when designing a successful test. These are: knowledge of the basic principles of the test and the correct choice of task system (Paylozyan \& Tadevosyan, 2009). The following requirements must be taken into account when designing a test:

- The test should be trustworthy. By saying trustworthy we understand that test results must be consistent regardless of the circumstances. In different circumstances, there should be almost no difference when different auditors apply the same test and evaluation results to different auditors.

- The test must be reliable. Reliability means that the test must serve its purpose, that is, it must test the knowledge and skills it was designed to test. Reliability test ensures success in achieving a specific goal. The test is reliable if the degree of difficulty corresponds to the level of readiness of the learner. Compliance means that for the average learner the test should be neither easy, nor too difficult. It should be of medium complexity. If the test is too easy or too difficult for the learner, it means the test is not reliable.

- The test must be effective. A test that measures the learner's knowledge, abilities and skills effectively is considered effective. In terms of content, a test is effective if its covers the key issues of the topic or topics. The test is effective if the level of difficulty of the test corresponds to the level of readiness of the learner.

- The test must be practical or feasible, by the side of technical, financial and time. When selecting a test, it is very important to choose the right types of tasks. Tests in the test should be arranged from simple to complex according to Benjamin S. Bloom's cognitive behavioral taxonomy. The following is a thought process from simple to complex.

In the complex structure of speech activity, there are distinct manifestations that comprise the phonetic, that is, the form of speech, the phonetic processes, the vocabulary and the grammatical structure (Kadevarek, 2011). Speech disorders can affect any of these components or their systems. Thus, some deficiencies relate only to extracurricular processes and are expressed by speech impairment without any accompanying manifestations, others affect the phonetic system of the language and are manifested not only by speech problems but also by poor vocabulary mastery, which results in and reading disorders. Along with all this, there is a group of disorders that encompass both phonetic and vocabulary systems, which are expressed by the general underdevelopment of all aspects of speech. 
Children's and adults' speech disorders early detection problem, prevention, as well as further correction are relevant not only speech therapy but also to related sciences. The use of systematic analysis principle of speech disorders allows to reveal the difficulties of formation this or that sides of speech. Early detection of possible deviations of both verbal and later written speech allows to correct them through pedagogical (speech therapeutic) skills. As a result of the analysis of professional literature, it has become obvious that the results of speech evaluation mostly depend on the process of speech evaluation: how speech evaluation is performed, under what circumstances, when, where and the most importantly what tools, methods and resources are used.

Standardized assessments are empirically developed evaluation tools with established statistical reliability and validity. A standardized test is one that requires all test takers to answer the same items/questions in the same way and that is scored in a standard or consistent way, thus making it possible to compare the relative performance of individuals or groups of individuals. There are two types of standardized assessment instruments: norm-referenced and criterionreferenced.

So let's try to discuss the types of standardized tests. Norm-referenced tests are standardized tests designed to compare and rank test takers in relation to one another. Using a norm-referenced test makes it possible to compare an individual's score(s) with those of a large group of statistically selected individuals - usually of the same age or grade level—who have already taken the test. A norm-referenced score is typically reported as a percentile ranking. For example, an individual who scores in the 90th percentile has performed as well as or better than $90 \%$ of other test takers of the same age or grade level (i.e., those in the norming group).

But there are criterion-referenced tests that are measure an individual's performance against a set of predetermined criteria or performance standards (e.g., descriptions of what an individual is expected to know or be able to do at a specific stage of development or level of education). In educational settings, criterion-referenced tests may be used to assess whether the student has learned a specific body of knowledge. It is possible, and in fact desirable, for an individual to pass or earn a perfect score on a criterion-referenced test (Kadevarek, 2011; Tatarova, 1999).

Speech research and assessment by a speech therapist are based on the following principles:

- Complex approach. According to this principle, it's necessary to carry out not only speech therapeutic, but also medical-psycho-pedagogical research. 
- Completeness and clarity. It's necessary to carry out the research of two types of speech: oral and written, aspects: impressive and expressive; and components.

- Individual approach. The research process should be appropriate to age, personal characteristics.

- Ongoing research, which assumes primary research and monitoring (Speech Therapy in practice, 2017; Paylozyan \& Tadevosyan, 2009).

- Revealing existing capabilities. During the research it should be revealed a person's (with speech disorder) verbal and non-verbal existing skills, so as to manage the speech therapeutic work based on it.

- The following stages of speech therapeutic research are distinguished:

- Preliminary (orientation):

○ the study of documents (medical, pedagogical),

0 the conversation with the child (with speech disorder) and his/her parents

○ personal data

○ medical history data (life, illness, psychophysical and verbal development)

$\circ$ information about verbal and social environment, upbringing, members of family and etc.

- Basic. During this main speech therapeutic research all aspects of speech are explored.

- Differentiation.

- Final.

Child's speech therapeutic research consists of three sections:

1. A thorough study of verbal and general mental development of dynamics and upbringing conditions based on the conversation with the parents and analysis of child's documents.

2. Speech therapeutic psycho-pedagogical research by detailed recording of child's verbal and non-verbal activity.

3. The analysis of all received data and pedagogical evaluation (Tadevosyan, 2006; Zhukova, 1994).

The child's medical-pedagogical complex research and his/her medical-pedagogical complex evaluation of mental-verbal development allow the pedagogue to understand the individual characteristics of each child. As a result of the analysis of modern foreign professional 
literature, it's become obvious that in abroad the speech therapeutic research is carried out in the following steps:

- interview with parent and collection of incident story,

- informal assessment - games, toys, books, experiments,

- use of standardized tests,

- meeting and discussion with parents or guardians.

During speech therapy, it's carried out study of the structure and mobility of speech apparatus, voice, breathing, speech rate and rhythm, impressive and expressive speech, writing and reading, and verbal features are emphasized. The pronunciation of sounds is examined apart in syllables, in words, in connected speech (through dialogue, picture naming, repetition of words, sentences). Phonetic listening is examined through the following tasks of phonetic perception, analysis, combination, and perceptions:

- to determine the first (last) sound of the word,

- to determine the presence (absence) of a sound in a word,

- to determine the number of sounds in a word,

- to divide the word into syllables

- to spell the words,

- to say a word that starts with a given sound, etc.

The syllable structure of a word is explored by the repetition of words having different syllable structure and sentences that they formed. When researching vocabulary, it's necessary to explore:

- the active and passive vocabulary,

- the use of different parts of speech,

- the knowledge of synonyms, antonyms,

- the name of the action,

- the attribute name,

- the ability to make generalizations.

The research of grammatical structure of speech:

- morphological aspect: singular and plural number, adjective, verb conjugation, verb conjugation time, verb tenses, word formation, 
- syntactic aspect: types of sentences, structure, existence of grammaticism.

The research of connected speech assumes a dialogue according to plot picture, ability to build story from the series of picture, story retelling, forming a story on given topic.

During the research of written speech, the technical and semantic aspects are examined:

- Reading Technique: by letters, syllables, words, fluent, stuttering, "guessing", "small scale", "slowdown" "mirror", omissions of words and syllables, substitutions, only correct reading of word start, confusion of letters looking similar, tracking errors of line reading.

- Understanding of the meaning of what is read: the main idea, metaphorical meaning, causeeffect relationships.

The copywriting, dictation, self-written work is examined. The presence of specific and non-specific errors is noted. The samples of written work are attached to the research card. The samples of written work are attached to the research card. The speech therapist also examines the child's play activities: general and soft mobility, target activities (drawing, plotting, appliqué and constructive activity), cognitive sphere, self-service and social adaption skills, as well as focusing on the eyes, emotional communication, stereotyped speech, movements and actions, responding to the interlocutor's speech, using the word as a means of communication, the presence or absence of echolalia. The research data is summarized by the speech therapist in a speech research card, indicating a brief psycho-pediatric profile of the child, the results of the oral and written speech tests.

The above mentioned are the general aspects of speech evaluation of speech therapeutic research, but the process of speech evaluation is changing because of any verbal disorder, age characteristics and other conditions.

The speech therapist carries out the research of the pronunciation of the sounds individually using special topic pictures.

For the research of the pronunciation of sounds the material should include subject pictures collection for different group of sounds. Selection of subject pictures is carried out so that the studied sound is at the beginning, middle and end of the word, as the pronunciation of the sound is different in different positions. Due to this method it's becoming possible to pronounce the group of sounds. For each position of the word sound the speech therapist chooses not less than three pictures, so as to hear and record how the child utters that sound. When choosing a material, it is necessary to take into account that in Armenian there are words that have pronunciation different 
from writing, for example, when uttering the word "upq" ("arj") the sound "2" (dz) is heard "₹" (ch $\left.=\left[\mathrm{t} \int\right]\right)$ and the word sounds as "unц" ("arch") and so on.

During the research of sound pronunciation, the speech therapist points out the absence, substitution, confusion and distortion of sound. If the child does not name the picture, the speech therapist suggests repeating the words after his/her pronunciation (reflective speech). If the child has a problem with the pronunciation of the pictures names sounds, but he/she can pronounce that sound separately, it means that it is necessary simply to practice that sound in words and phrases.

During the speech research the speech therapist pays attention to the speech rate, clarity, the correct pronunciation of words, the sound of voice. If there are any deficiencies, they are noted in the speech cards. When conducting an individual survey, the speech therapist is writing down all the answers on the speech card, indicating the date of the survey (Logopediya, 2018).

\section{METHODOLOGY}

The methodology of information gathering, processing and analysis research is based on a quantitative approach that allows to combine quantitative data collection by using quantitative methods, subsequently being able to draw conclusions from the data, based on established numerical patterns (Tadevosyan, 2006; Tatarova, 1999). The strength side of quantitative research is that no matter how many researchers do it, they would all come to the same / similar conclusions, as the methods and methodologies of revealing social reality are accurate. The following methods were used within the frame of current study:

- Study of the standardized tests usage of speech research in contemporary speech therapy.

- Study of specialized professional literature and theoretical analysis.

- Analysis of children's pedagogical documents in public education.

- Study of speech cards and medical history data, inquiry and testing.

- Tests

- Generalization of results, summarizing qualitative and quantitative data.

- Experimental study.

- Combination of data of experimental study.

- Analysis of mathematical, statistical data (Tatarova, 1999).

The strength side of the qualitative research, in contrast to quantitative research, is that the more a researcher studies the same problem, the more detailed and in-depth information will be 
obtained. That is, qualitative research requires a research style, largely influenced by the researcher's outlook and research preferences. If in a case of quantitative research, we introduce the reliability of research, then in qualitative research we introduce the harmony of research and its being reasonable and convincing. The quantitate questionnaire was developed online and the data was collected online. The test was formed with the help of an online program which allowed more clearly to receive information of qualitative and quantitative analysis data. That test was conducted to analyze the objectivity of the speech research process in Armenia. The test included the following questions: Profession of the responded; The type of activity; Do you find the speech research process important in your work? Do you have any tool to carry out speech research process? What do you think the speech research that carried out by you can be considered objective? Do you use any standardized tests for speech research? Please indicate the tools, methods, resources and standardized tests, which you used in speech research, evaluation process. Can the use of standardized tests promote the efficiency of the speech evaluation process? How would you like a standardized test of speech evaluation relevant to native language to be?

\section{Participants}

The target group of the research is 31 speech therapists and related field specialists: psychologists and art therapists of different ages, from different institutions, cities, villages, regions of Republic of Armenia. They were interviewed using special formed test. Below is the brief information of the research participants (Table 1), as well as their specialization and activities (Table 2).

Table 1. Research participants

\begin{tabular}{|l|l|l|l|l|l|l|}
\hline \multicolumn{2}{|l|}{ Gender distribution } & \multicolumn{3}{l|}{ City distribution } \\
\hline male & female & Yerevan & Stepanavan & Vardenis & Martuni & Sevan \\
\hline 1 & 30 & 27 & 1 & 1 & 1 & 1 \\
\hline
\end{tabular}

Table 2. The activities of the research participants

\begin{tabular}{|l|l|l|l|l|l|l|}
\hline \multicolumn{2}{|l|}{ Type of activity } & \multicolumn{3}{l|}{ Specialization } \\
\hline Student & Lecturer & $\begin{array}{l}\text { Practical } \\
\text { worker }\end{array}$ & $\begin{array}{l}\text { Speech } \\
\text { therapist }\end{array}$ & Psychologist & $\begin{array}{l}\text { Special } \\
\text { educator }\end{array}$ & Art therapist \\
\hline 3 & 1 & 27 & 26 & 3 & 1 & 1 \\
\hline
\end{tabular}


Before filling the test each of the research respondents were provided with the information about the purpose and relevance of research, in order to obtain their consent and permission, to engage themselves and their children as a research participant.

\section{Data analysis}

The results of data analysis are:

- $100 \%$ of the respondents mentioned the importance of word research, evaluation process in their work. It should be emphasized that not only the speech therapists, but also psychologists, special educators and art therapists participated in the research and they also gave importance to the process of child's speech assessment.

- $81.5 \%$ of the respondents stated that they had any tools, methods or recourses to carry out speech research. Only $18.5 \%$ of the respondents stated that they did not have a specific tool, method or recourse for this process. However, it should be noted that the last group participates are not speech therapists and the process of speech evaluation is not priority for them (Picture 1).

Picture 1. The availability of tools and methods of speech evaluation

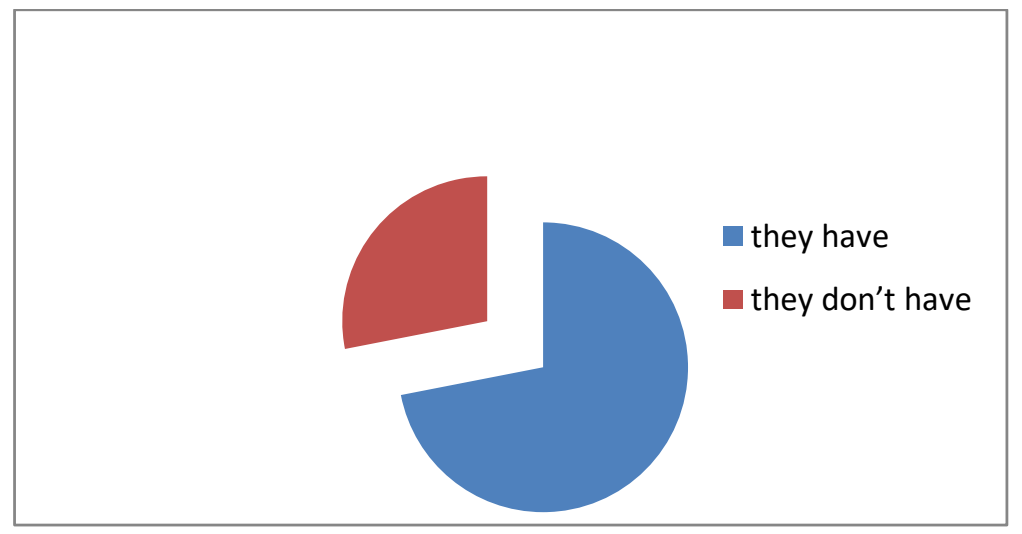

- It seems to be a normal process when a specialist for whom the speech evaluation process is not a priority does not have the tools and resources for speech evaluation. However, $31 \%$ of the respondents find that the speech assessment process is subjective and it cannot be considered objective, so other speech therapists may disagree with the diagnosis given by him/her or the course of his/her developed work. It is a worrying indicator. And $72.4 \%$ of the respondents find out that their research and results are objective. 
- It is believed that if there were common tool, method, resource and standardized test, then the objectivity of the word research and evaluation process would be ensured. However, only $17.2 \%$ of respondents mentioned that they use standardized tests, but none of them mentioned the exact test names so we cannot analyze how objective the test results will be. And $82.8 \%$ of respondents do not use any clearly standardized tests, even the majority of them mentioned that they use tools, methods and resources developed by them or they combine several methods to obtain reliable information. The question arises, is the objectivity provided in this case?

- $82.1 \%$ of the respondents consider that the use of standardized tests of speech research can increase efficiency of this process and will ensure the objectivity of the process. $3.6 \%$ of respondents answered no, 3.6\% partially, 3.6\% situational, 3.6\% linked the efficiency of standardized tests to the features of speech disorder, $3.6 \%$ consider it was possible.

- Some answers of the following question "How a corresponding native language standardized test of speech research and evaluation should be" are introduced below:

○ Multi-profile.

○ Detailed, coordinated.

- To be formed based on the typical development of the speech.

- To be available and useful for the relating field specialists.

○ With simple, clear formulations.

- To be consist of simultaneous inclusion of phonetic-sound, sound-syllable, vocabularygrammar aspects.

$\circ$ I find it difficult to answer.

- It's a global issue.

- Quite detailed and complex.

- Easy to understand, objective, adopted to the age of child.

- Dedicated to simple points that would be more visible in practice. I would like to be provided with hinted answer for the specialist and with the analyses of assessment levels of the problem (middle, easy problem, based on).

○ Colorful, illustrated, easy and short.

○ Comprehensive and scientifically-practically tested and proofed.

○ I can't say for sure, yet. 
- As simple as possible also for the use of psychologists (but also difficult and accurate so as to trust the results).

○ Reliable, trustworthy and measurable.

- Multifunctional and detailed according to the group age and the level of development.

○ Practical

○ Questions from maternal pregnancy, heredity to a child's specific age should be included in the test.

- In order to really match the native language and be without exaggeration.

\section{RESULTS}

The analysis of relevant sources allows us to conclude that in the process of diagnostics and evaluation, speech therapy standardized tests are used in the United States and a number of European countries, however in the Russian Federation standardized tests are not used. They use tests which promote speech research process, but they are not standardized and cannot give reliable and objective assessment results. However, it should be stated that a number of international organizations of our country, Republic of Armenia tend to take the standardized tests of the US and a number of European countries and translate and use them. This has its disadvantage, as it will not reflect the peculiarities of the Armenian language and it will not take into account the educational standards of the Republic of Armenia, so it cannot be useful for specialists in organizing the speech therapeutic research process.

Thus, according to our hypothesis, in the process of speech therapeutic evaluation and research the use of standardized tests will ensure objectivity and will allow to obtain complete and reliable information about the research participant and his/her speech.

Taking into account the above mentioned, we developed a special test for specialists, which allowed us to find out the opinions of specialists working in the Republic of Armenia about speech research and evaluation process.

The results of the test show that all the participates value the word evaluation process in their work, majority of them have some tools, methods and resource that help them carry out speech research, but the other do not have a specific tool, method and resource for this process. It is worrying indicator that a group of specialist considers that the process of evaluating speech, carried by them, is subjective and cannot be considered objective, so other speech therapists may 
disagree with the diagnosis or the course of his/her developed work. The majority of respondents find that their research and results are objective and reliable. It should be emphasized that some specialists use standardized speech test, but none of them gives exact test names, so we cannot analyze how objective the test results will be, but at the same time, the majority of does not use any clearly standardized test, even some of them mentioned that they use tools, methods and resources developed by them or they combine several methods to obtain reliable information. And as we thought at the beginning of the study, majority of practice field specialists of the Republic of Armenia find that the use of standardized tests can ensure the objectivity evaluation and diagnostics of speech research, as a result we there will be reliable and trustworthy data, and the specialist will ensure the process of his or her word research. And surely, as a result, we will have a correct and coordinated corrective-development process of speech therapy. It is stated that the more reliable and objective the results of the speech evaluation process are, the more correct the correction process is organized. As a result, the working process of speech therapy becomes more effective. Thus, the process of successful speech research is one of the keys of the success of speech therapy.

\section{DISCUSSION}

Based on RA Government protocol decision of Session N1 on January 9, 2014, “A person's comprehensive evaluation of World Health Organization International Classification of Functioning based on Implementation model of defining disability (hereinafter WHO ICF-CY)" according to which in recent years, in Republic of Armenia, in the scope of the social model holding of defining disability, improvements are carrying out not only in the area of defining disability but also in areas of disability evaluation, disability grade provision, service creation and provision, monitoring, and evaluation (Government of the Republic of Armenia 2014 Annex N 1 to the Protocol on the January 9 Session Decision Protocol Annex 1: Introduction of a Model for Defining Disability Based on the International Classification of Functions of the World Health Organization).

As already mentioned in this work, the WHO ICF-CY allows the use of common language and terminology, thereby recording the problems in organ system or in its functions, limitations of person's activity and participation, as well as the impact of corresponding environmental factors in the period of neonatal, childhood and adolescence (Avetisyan, Ghazaryan \& Malkhasyan, 
2017). Functional evaluation allows receiving comprehensive, coordinated information about the person who is evaluated. However, our research allowed us to come to the conclusion that during the functional evaluation objective, reliable and trustworthy information, tools, methods and recourses of speech research cannot be used in the Republic of Armenia.

We also came to the conclusion that it is necessary to create standardized test which will allow to do objective evaluation of speech. After the test is created and tested, it will be necessary to pass an independent examination; it means that the best specialists will be involved in this process, to get the best product. But we have to remember that standardized tests are limited however when it comes to planning effective oral and written language intervention. Other, more specific, assessments can be utilized to plan for intervention.

\section{DISADVANTAGES OF THE WORK}

- One of the disadvantages of the work is the number of respondents. 31 specialists, practitioners took predication in survey. It should be noted that the location of the research includes not only Yerevan but it also the other regions of the Republic of Armenia. We think that if we could involve more specialists we would have more objective information.

- The chosen methodology is limited, it was necessary not only to satisfy one diagnostic test but also to develop and use other examples of tests to compare the data obtained.

- Lack of relevant sources. Since, many countries do not have standardized tests for speech research, so there is a lack of relevant sources.

\section{PRACTICAL AND SCIENTIFIC RECOMMENDATIONS}

- To perform a more detailed analysis of the bibliography.

- To invite a roundtable discussion, to bring together professionals who value the speech evaluation process in their professional work

- To discuss the specifics of the features of speech research and evaluation process, during the roundtable discussion.

- To emphasize the problems which a specialist faces when evaluating speech.

- To create an applicable test for speech research and evaluation.

- To use, practice the created test. 
- To improve and develop the created test.

- To use already developed and approved by professionals' test for more than 5000 people.

- To standardize the test.

\section{CONCLUSION}

Thus, according to our hypothesis, the use of standardized tests in the process of speech therapeutic evaluation and research will ensure objectivity and will allow to obtain complete and reliable information about the research participant and his/her speech. Our hypothesis is confirmed by our research participants. It is necessary to create speech evaluating diagnostic test corresponding to native language and educational standards of Republic of Armenia and which will be used and we will already receive reliable data on to what extent the use of standardized tests of speech research will promote the efficiency of speech therapy, ensure the objectivity of the speech evaluation process and provide reliable and trustworthy results.

\section{REFERENCE LIST}

1. Association of Language Testers in Europe (2014), https://www.alte.org/

2. Avetisyan, M., Ghazaryan, T., Malkhasyan, A. (2017) Hashmandamutyun unecogh 2-18 tarekan erekhaneri kensagorcuneutyan, masnakcutyan ev mijavayrayin gwrtsonneri gnahatman metodakan dzernark (Methodological Manual for Assessing the Functional Activity, Participation and Environmental Factors of 2-18 Years Old Childrenwith disabilities), Yerevan, Zangak

3. HH karavarutyan 2014t. hunvari 9-i nisti N 1 ardzanagrayin voroshman hayecakarg Havelvac $\mathrm{N}$ 1: andzi bazmakoxmani gnahatman arokhchapahutyan hamashkharhayin kazmakerputyan funkcianeri mijazgayin dasakargman skzbunqneri vra himnvac hashmandamutyan sahmanman modeli nerdrman (Government of the Republic of Armenia 2014 Annex N 1 to the Protocol on the January 9 Session Decision Protocol Annex 1: Introduction of a Model for Defining Disability Based on the International Classification of Functions of the World Health Organization)

4. Individuals with Disabilities Education Improvement Act of 2004, Sections 300.301300.305 
5. Gromova, O. E. (2003) Metodika formirovaniya nachalnogo detskogo leksikona v usloviyakh napravlennogo korrekcionno-razvivayushego obucheniya (Methodological formulation of preliminary childish lexicon in terms of corrective-developmental education): dis. Kand. Ped. Nauk 13.00.03. Moskva.

6. Kaderavek, J. N. (2011) Language Disorders in Children: Fundamental Concepts of Assessment and Intervention, Allyn \& Bacon

7. Logopedian gorcnakanum (Speech Therapy in practice) (2017) / A. V. Avagyan ev urishner, Yerevan, Asoghik, ej 19

8. Logopedia. Teoriya i praktika (Speech therapy, Theory and practice) (2018), Moskva, "Eksmo"- 608 s.

9. Miqaelyan, O., Miqaelyan, S. (2010) Yntaciq gnahatman nor hamakargy vorpes krtutyan voraki barelavman mijoc (New system of on-going assessment as a means of improving the quality of education), Yerevan

10. Paylozyan Zh. H., Tadevosyan, E. R. (2009) Khosqayin nyuter jisht hnchartaberman amrapndman hamar (Speech materials to enhance right sound performance), Yerevan, "Yerevani Anania Shirakatsu anvan jemaran krtahamalir" PBY, 78 ej

11. Tadevosyan, G. (2006) Vorakakan swcialakan hetazotutyunner (Qualitative social research), Yerevan, EPH

12. Tatarova, G. (1999) Metodologiya analiza dannikh v sociologii (Data Analysis Methodology in Sociology), M., "Institut Otkritoe Obshestvo".

13. Westby, C., Burda, A., \& Mehta, Z. (2003) Asking the right questions in the right ways: Strategies for ethnographic interviewing. The ASHA Leader.

14. World Health organization (2001) International Classification of Functioning, Disability and Health (ICF) Retrieved from URL www.who.org

15. Zhukova, N. S. (1994) Preodolenie nedorazvitiya rechi u detey (Overcoming Speech Underdevelopment in Children) Uchebno-metod. Posobie - Moskva: Soc.-polit., zhurnal, $96 \mathrm{s.}$ 